\title{
Laser cleaning of diagnostic mirrors from tokamak-like carbon contaminants
}

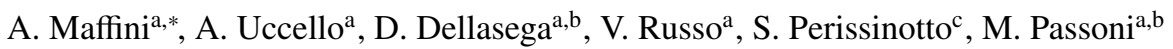 \\ ${ }^{a}$ Dipartimento di Energia, Politecnico di Milano, Milan, Italy \\ ${ }^{b}$ Istituto di Fisica del Plasma, Consiglio Nazionale delle Ricerche, EURATOM-ENEA-CNR Association, Milan, Italy \\ ${ }^{c}$ Center for Nano Science and Technology @ Polimi, Istituto Italiano di Tecnologia, Milan, Italy
}

\begin{abstract}
This paper presents a laboratory-scale experimental investigation of laser cleaning of diagnostic First Mirrors (FMs). Redeposition of contaminants sputtered from tokamak first wall onto FMs surface could dramatically decrease their reflectivity in an unacceptable way for the functioning of the plasma diagnostic systems. Laser cleaning is a promising solution to tackle this issue. In this work, pulsed laser deposition was exploited to produce rhodium films functional as FMs and to deposit onto them carbon contaminants with tailored features, resembling those found in tokamaks. The same laser system was also used to perform laser cleaning experiments by means of a sample handling procedure that allows to clean some $\mathrm{cm}^{2}$ in few minutes. The cleaning effectiveness was evaluated in terms of specular reflectivity recovery and mirror surface integrity. The effect of different laser wavelengths $(\lambda=1064,266 \mathrm{~nm})$ on the cleaning process is also addressed.
\end{abstract}

Keywords:

Carbon contamination, Redeposition, Laser ablation, Reflectivity, Tokamak

\section{Introduction}

Metallic First Mirrors (FMs) will be crucial components of all optical systems for plasma diagnostics and imaging tools in ITER [1]. Redeposition of sputtered materials from the first wall onto FMs surface is one of the main concerns that affects their performance. Thanks to its accurate focusing, insensitivity to magnetic fields and the possibility of remote operation, laser cleaning is a potential solution for the in-situ cleaning of FMs in ITER and future fusion machines. To date, some promising results have been achieved in preliminary laser cleaning experiments of mirrors exposed to tokamak plasmas [2-4]. Despite this approach is clearly of primary interest, it does not allow to directly control the nature of the contaminant and hence to evaluate the impact of its characteristics on the physics of the cleaning process. A complementary solution is to exploit suitable deposition techniques to simulate the process of contaminants redeposition on FMs at laboratory-scale. The possibility to obtain ad hoc prepared samples with controlled features may provide a significant benchmark for laser cleaning of different materials, which could in principle show a different response to laser irradiation. This aspect is particularly important considering that the exact nature of the redeposits in ITER and future machines is unknown. In [5], we developed a novel, lab-scale approach in which Pulsed Laser Deposition (PLD) is exploited to produce rhodium $(\mathrm{Rh})$ mirrors $[6,7]$ and to deposit properly designed carbon $(\mathrm{C})$ contaminants onto them. The same laser source is

*Corresponding author at: Dipartimento di Energia, Politecnico di Milano, Via Ponzio 34/3, 20133, Milan, Italy. Tel.: +39 0223996354; Fax: +39 0223996309.

Email address: alessandro.maffini@polimi.it (A. Maffini) successively used to perform the cleaning procedure. Together with other metals like tungsten, copper and molybdenum, $\mathrm{Rh}$ is one of the principal candidate materials for ITER FMs [8]. C is a well-investigated plasma facing material, and has long been considered for the ITER divertor design. Despite the recent decision to switch to an all metal ITER first wall design, $\mathrm{C}$ is still employed in some of the operating fusion research machines and some of the preliminary experiments about laser cleaning for fusion application have dealt with $C$ removal $[9,10]$. In [5] the investigation was limited to a single typology of $\mathrm{C}$ contaminant, and the cleaning procedure therein exploited only allowed the cleaning of a small portion of the mirrors. In this work, the laser cleaning of $\mathrm{Rh}$ mirrors from tokamak-like $\mathrm{C}$ deposits is discussed, to develop a suitable procedure relevant for tokamak application. The peculiar PLD capability of tailoring deposit features at the nanoscale was exploited to obtain two very different kinds of $\mathrm{C}$ contaminants, i.e. compact films and porous film with an open morphology, that can be regarded as representative of the wide variety of $\mathrm{C}$ redeposits in a tokamak. Laser cleaning experiments were performed in vacuum, with a sample handling procedure that allows to treat areas of some $\mathrm{cm}^{2}$ in a few minutes. Laser parameters were chosen in order to guarantee the integrity of the reflective surface. Two different laser wavelengths were employed and their effect on the cleaning process was addressed. The cleaning effectiveness was evaluated by reflectivity measurements. Surface modifications of the samples after laser irradiation were investigated by Scanning Electron Microscopy (SEM). In section 2, the preparation and characterization of $\mathrm{C}$ deposits on $\mathrm{Rh}$ films, together with a comparison to the redeposition processes in tokamaks, will be described. The laser cleaning procedure will be presented in 
section 3 , and the cleaning results will be shown and discussed in section 4. Concluding remarks will be given in section 5 .

\section{Materials and methods}

Our PLD apparatus exploits a Q-switched Nd:YAG laser with a tunable wavelength $(\lambda=1064,532$ and $266 \mathrm{~nm})$, pulse duration 5-7 ns and repetition rate $10 \mathrm{~Hz}$. The laser beam has a circular shape of $\varnothing=9 \mathrm{~mm}$. Laser pulses hit the target of the material to be deposited in a high-vacuum chamber $\left(3 \times 10^{-3} \mathrm{~Pa}\right)$, causing the evaporation of its superficial layers. The ablated species impinge on a suitable substrate placed at a distance $\mathrm{d}_{\mathrm{T}-\mathrm{S}}$ from the target. The deposited films were characterized, before and after laser exposure, with a Zeiss Supra 40 field emission SEM (accelerating voltage 3-6 kV) to assess their surface morphology and with a Renishaw InVia Raman spectrometer using the $514.5 \mathrm{~nm}$ wavelength of an $\mathrm{Ar}^{+}$laser to determine the feature of $\mathrm{C}$ deposits. To check the film elemental composition, we performed energy dispersive $\mathrm{x}$-ray spectroscopy (EDXS). In addition, the specular reflectivity $\left(R_{\text {spec }}\right)$ of the $\mathrm{Rh}$ mirrors before and after cleaning was determined subtracting the diffuse reflectivity to the total reflectivity, both measured by a UV-vis-NIR PerkinElmer Lambda 1050 spectrophotometer with a $150 \mathrm{~mm}$ spectralon integrating sphere on the $260-1800 \mathrm{~nm}$ wavelength range, of interest for most of the ITER diagnostics.

\subsection{Rh films deposition}

Among the possible nanostructures obtainable for $\mathrm{Rh}$ films by means of PLD [6], the highly-oriented polycrystalline one (preferential growth direction (111)) [7] was chosen, mainly because of its high adhesion to the substrate which could guarantee a high laser damage threshold. Rh films $\sim 1 \mu \mathrm{m}$ thick were produced on a $2.6 \times 2.6 \mathrm{~cm}^{2}(100)$ silicon substrate. Details are given in [7].

\subsection{C contaminants deposition}

The production of PLD contaminants was directed towards the realization of $\mathrm{C}$ deposits similar to those found in nowadays tokamaks [11-14]. Their properties depend on both the specific characteristics of the tokamak and the position within the first wall where the deposit builds up. They can exhibit different properties in terms of morphology (e.g. compact layers, porous films, dust), nature of C-C bonds and hydrogen content [11]. Most of these features are related to the energy of the impinging species reaching the growing deposit. High energy $\mathrm{C}$ ions (hundreds of $\mathrm{eV}$ ) lead to hard, dense, $s p^{3}$ rich films, while low energies are typical of disordered graphitic nanoclusters assembled in porous structures $[12,13]$. By properly choosing the PLD parameters we produced both compact films and porous films with an open morphology, which are representative of the wide variety of tokamak C contaminants. A $99.9 \%$ purity graphite target was ablated by 3000 laser pulses with $\lambda=532 \mathrm{~nm}$. The laser fluence per pulse on the target was $1.6 \mathrm{~J} / \mathrm{cm}^{2}$. By means of a helium (He) atmosphere it is possible to tune the energy of the $\mathrm{C}$ species impinging the $\mathrm{Rh}$ film. The higher is the He pressure and the larger is the $d_{T-S}$, the lower is the energy of the species.
Compact, flat, featureless films $\sim 300 \mathrm{~nm}$ thick were obtained with $5 \mathrm{~Pa}$ of $\mathrm{He}$ and $d_{T-S}=7 \mathrm{~cm}$. Increasing the He pressure to $60 \mathrm{~Pa}$ and $d_{T-S}$ to $9 \mathrm{~cm}$ the kinetic energy of the $\mathrm{C}$ species is low enough to allow the formation of porous films with open morphology and an average thickness of $\sim 2 \mu \mathrm{m}[5,15]$.

The $\mathrm{C}$ films were characterized by Raman spectroscopy. The main features of $C$ Raman spectra are the $G$ peak (in the range $\left.1500-1630 \mathrm{~cm}^{-1}\right)$, due to stretching of $s p^{2} \mathrm{C}-\mathrm{C}$ bonds, and the disorder-activated $\mathrm{D}$ peak $\left(\sim 1350 \mathrm{~cm}^{-1}\right)$, related to the breathing mode of sixfold aromatic rings [16]. Figure 1 shows the Raman spectra of compact (a) and porous (b) C films. Considering the $\mathrm{I}(\mathrm{D}) / \mathrm{I}(\mathrm{G})$ intensity ratio and the $\mathrm{G}$ peak position, it is possible to estimate the degree of graphitic order and the $s p^{3}$ content of the films [16]. Compact films can be regarded as amorphous carbon, with an almost complete loss of crystalline order and a small fraction $(\leq 15 \%)$ of $s p^{3}$ bonds. Porous $\mathrm{C}$ films are made of a nearly pure $s p^{2}$ network of disordered, nanometric graphitic domains [15]
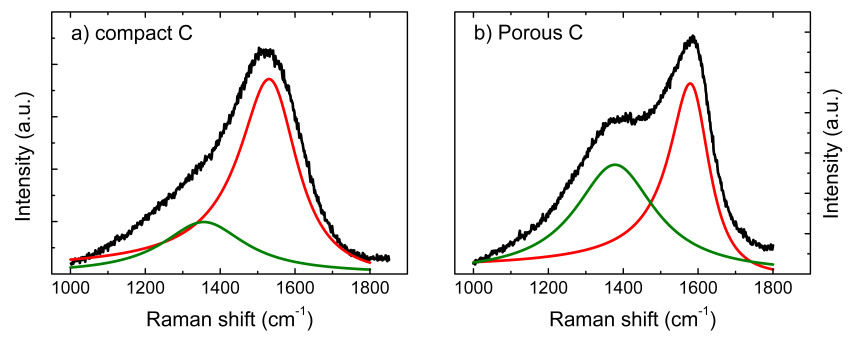

Figure 1: Raman spectra of PLD compact (a) and porous (b) $\mathrm{C}$ together with fitting curves for D (Lorentzian, green) and G peaks (Breit-Wigner-Fano, red).

To allow the subimplatation of $\mathrm{C}$ ions, a process necessary to trigger the formation of $s p^{3}$ bonds in the growing film, a threshold energy of $32 \mathrm{eV}$ per $\mathrm{C}$ ion is needed [17]. Considering PLD deposition, it was found that $\mathrm{C}$ ions with an average energy of $\sim 50 \mathrm{eV}$ leads to $\sim 35 \%$ of $s p^{3}$ bonds [18]. Our C compact films could therefore originate from $\sim 40 \mathrm{eV} \mathrm{C}$ ions. The porous films arose instead from the aggregation of nanoclusters with energies on the order of the eV [15], typical of particles in regions shadowed from the main plasma [1]. The results here reported for $\mathrm{C}$ could be easily extended to other tokamak relevant materials. PLD is extremely versatile and offers a fine control of the morphology and structure of the deposited materials. It could thus represent an ideal tool to simulate the effect of contaminant redeposition in tokamaks.

\section{Laser cleaning procedure and process parameters}

To carry out the laser cleaning experiments, the PLD apparatus was modified, replacing the target with the sample to be cleaned. Laser cleaning was performed in high-vacuum $(3 \times$ $10^{-3} \mathrm{~Pa}$ ) to avoid oxidation and to consider realistic conditions in view of tokamak application. The laser beam hits the sample at $45^{\circ}$, and the laser spot on the sample has an elliptical shape with the major axis of $\sim 13 \mathrm{~mm}$. Two different laser wavelengths were exploited: $1064 \mathrm{~nm}$ (Near InfraRed, NIR) and $266 \mathrm{~nm}$ (UltraViolet, UV). NIR wavelength is well suitable for optical 
fibers, facilitating remote handling. UV pulses were exploited to enhance the performance of the process. A suitable laser cleaning procedure must guarantee the integrity of the surface to be treated; therefore, the laser fluence must be chosen accordingly. In [5] we previously determined the NIR laser damage threshold for the highly-oriented Rh films. A laser fluence of $350 \mathrm{~mJ} / \mathrm{cm}^{2}$, which did not yield any damage of the Rh film even for 3000 pulses per site, was chosen also in this work. Since the laser damage threshold of Rh films should increase with lowering $\lambda$ [19], in the case of UV pulses we employed the maximum fluence obtainable with our laser source, which is close to $150 \mathrm{~mJ} / \mathrm{cm}^{2}$.

With the aim of cleaning areas of some $\mathrm{cm}^{2}$ ensuring a uniform irradiation of the sample, the latter was moved during the cleaning. We developed a sample handling procedure that allows a fast treatment and it is easily scalable to samples of arbitrary size. A sketch of the "snake pathway" described by the laser spot is represented in figure 2. The sample is moved with

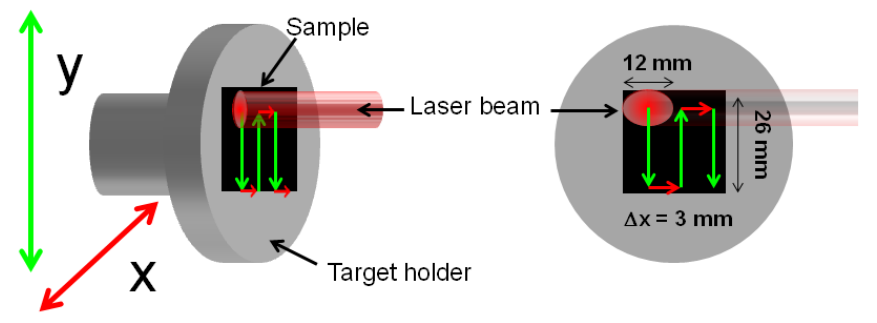

Figure 2: Scheme of the "snake pathway" procedure. The path of the laser spot is highlighted with arrows on the sample.

a selected vertical velocity $\mathrm{v}_{\mathrm{y}}$. At the end of the stripe the laser is shut down and the sample is moved forward by a step $\Delta x$. Then, another vertical cleaning stripe, in the opposite way, is performed. The procedure can be repeated until all the surface is treated. From preliminary cleaning experiments we found that $\mathrm{v}_{\mathrm{y}}=4 \mathrm{~mm} / \mathrm{s}$ and $\Delta x=3 \mathrm{~mm}$ ensure a good compromise among cleaning effectiveness, time requirement and integrity of the substrate. With these parameters, the cleaning time of a $2.6 \times 2.6 \mathrm{~cm}^{2}$ sample is less than 5 minutes and the number of pulses per site is $\sim 92$, well below the damage threshold for both NIR and UV. Visual inspection and SEM analysis confirmed that Rh mirrors handled with the snake-pathway procedure and irradiated with $1064 \mathrm{~nm}$ laser pulses at $350 \mathrm{~mJ} / \mathrm{cm}^{2}$ or with $266 \mathrm{~nm}$ laser pulses at $150 \mathrm{~mJ} / \mathrm{cm}^{2}$ did not show any damage.

\section{Cleaning results}

The effectiveness of the laser cleaning procedure was evaluated in terms of the specular reflectivity $\left(R_{\text {spec }}\right)$ of the samples. Figure 3 shows $R_{\text {spec }}$ of a Rh film as deposited (line I), contaminated with a compact $\mathrm{C}$ film (line II) and after a single scan with NIR pulses (line III). Comparing lines II and III, a very satisfactory $R_{\text {spec }}$ recovery for the whole wavelength range is evident. The SEM analysis (not reported here) showed that the
$\mathrm{C}$ film was removed and the Rh mirror was not damaged. The presence of micrometric, not fully ablated $\mathrm{C}$ debris suggests that thermal stresses arising in the compact $\mathrm{C}$ film during laser heating may play a major role in its removal.

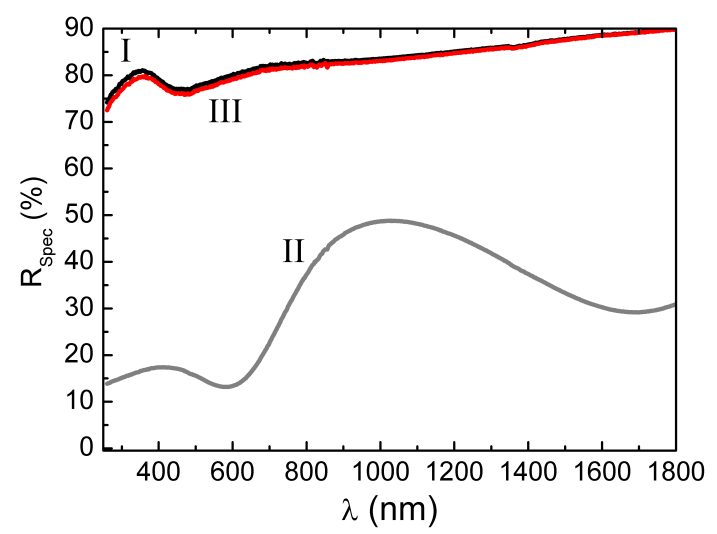

Figure 3: Laser cleaning of Rh mirrors from compact $\mathrm{C}$ contaminants. $R_{\text {spec }}$ of: I) As dep. Rh films II) Compact C film III) Rh film after cleaning.

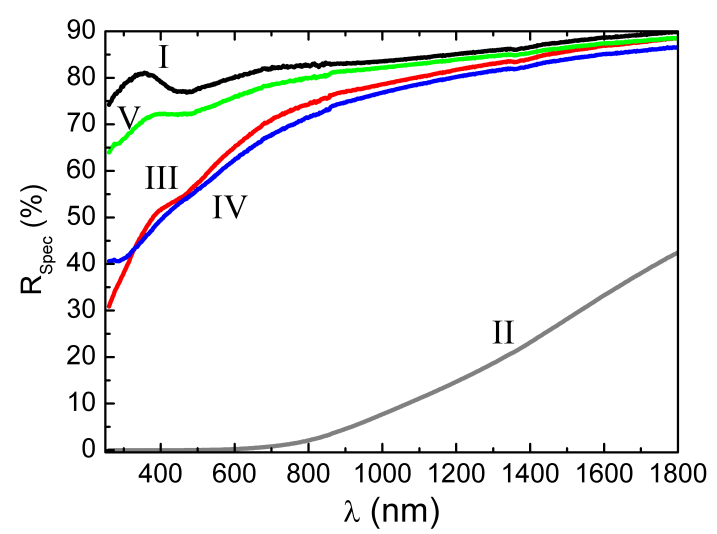

Figure 4: Laser cleaning of $\mathrm{Rh}$ mirrors from porous $\mathrm{C}$ contaminants. $R_{\text {spec }}$ of: I) As dep. Rh films II) Porous C film III) After IR cleaning IV) After UV cleaning V) After cleaning with IR (first scan) plus UV(second scan).

The cleaning results related to the porous $\mathrm{C}$ quasi-films are reported in figure 4 . By comparing $R_{\text {spec }}$ of the $\mathrm{Rh}$ film as deposited (line I) and after a single scan with NIR pulses (line III), one can observe that loss in $R_{\text {spec }}$ is below $10 \%$ for $\lambda>700 \mathrm{~nm}$. However, approaching the visible and UV range, $R_{\text {spec }}$ dramatically decreases $\left(R_{\text {spec }}=30 \%\right.$ at $\left.\lambda=260 \mathrm{~nm}\right)$. Further attempts to increase the cleaning effectiveness were performed with additional laser scans using the same $\lambda$ and fluence. The $R_{\text {spec }}$ increase was still modest, especially in the UV region. Figure 5 shows two SEM top view images of a porous $\mathrm{C}$ sample after a single NIR scan. Rh mirror maintained its integrity, but the presence of trail-shaped residuals can be appreciated (figure 5a). The trails are mainly made of $\mathrm{C}$, as revealed by EDXS analysis (not shown). The spacing between two adjacent trails is equal to the distance traveled by the sample between two subsequent pulses. This suggests that they were formed at the edge of the laser spot, where the fluence was not high enough to fully remove the $\mathrm{C}$ deposit but still sufficient to change the properties 
of the remaining material, making it much more resistant to ablation. Raman analysis reveals a $s p^{2}$ clustering process toward nanocrystalline graphite as a consequence of laser irradiation, as can be deduced from a shift of the $G$ peak to $\sim 1600 \mathrm{~cm}^{-1}$ and an increasing of $I(D) / I(G)$ ratio to 1 (not shown) [16]. Since the measured diffuse reflectivity is negligible (less than
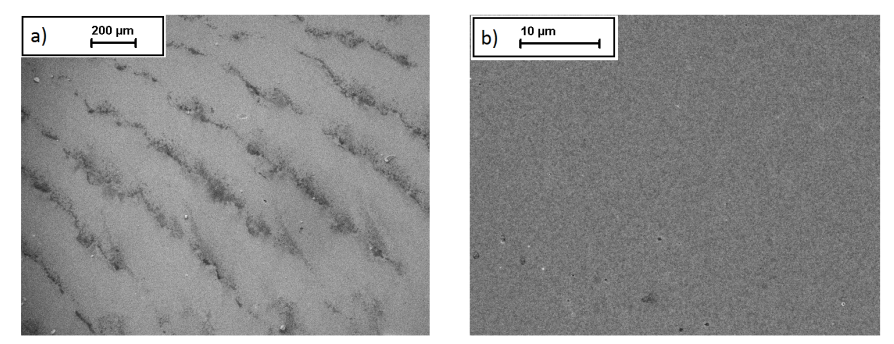

Figure 5: SEM top view of porous $\mathrm{C}$ on Rh after NIR cleaning (a) 200x magnification. Trails are appreciable. (b) 5000x magnification in the area between two trails. No C residuals are present.

$2 \%$ ), the loss in $\mathrm{R}_{\text {spec }}$ is mainly due to light absorption by $\mathrm{C}$ residuals. Line III of figure 4 shows a drop of $\mathrm{R}_{\text {spec }}$ at $\lambda=1064$ $\mathrm{nm}$ of only $5 \%$, meaning that the absorption of NIR light by the residuals is low. This could explain the reduced cleaning effectiveness of the NIR scans after the first one. The minimum of $\mathrm{R}_{\text {spec }}$ in the $\mathrm{UV}$ region indicates a stronger absorption of the $\mathrm{UV}$ light by $\mathrm{C}$ residuals.

Cleaning experiments with $\lambda=266 \mathrm{~nm}$ and fluence 150 $\mathrm{mJ} / \mathrm{cm}^{2}$ (see sec. 3) were therefore carried out. $R_{\text {spec }}$ of a $\mathrm{Rh}$ mirror contaminated with the porous $\mathrm{C}$ quasi-film after a single UV scan is reported in figure 4, line IV (blue). As expected, $266 \mathrm{~nm}$ pulses gave a better $\mathrm{R}_{\text {spec }}$ recovery in the UV region than $1064 \mathrm{~nm}$ pulses (line III). However, for $\lambda>350 \mathrm{~nm}$, NIR cleaning performed slightly better. The $\mathrm{R}_{\text {spec }}$ recovery for the two wavelengths was substantially equivalent. It should be anyway underlined the significant difference of the laser fluence in the two cases. Unlike what observed after a NIR scan, at a visual inspection the UV cleaned Rh mirror showed a brownish halo, but the presence of trails was less evident. From this point of view, the two wavelengths seem to be complementary.

Following this consideration we took advantage of the combined effect of NIR and UV pulses. As a first step, the porous C contaminated Rh mirror was treated with $1064 \mathrm{~nm}$ pulses which ensure an effective removal of most of the contaminant. Then, in order to remove the UV absorbing $\mathrm{C}$ residuals, a further scan with $266 \mathrm{~nm}$ pulses was performed. Thanks to this procedure, the extent of the mirror surface covered by the trails is strongly reduced, as results from a visual inspection. Line $\mathrm{V}$ in figure 4 shows the $\mathrm{R}_{\text {spec }}$ after the NIR $+\mathrm{UV}$ cleaning. The loss of $\mathrm{R}_{\text {spec }}$ in the range of 450-1800 $\mathrm{nm}$ varies between $1-5 \%$, and even in the UV region it does not exceed $12 \%$. A further enhancement of $\mathrm{R}_{\text {spec }}$ recovery, can be expected by repeating this cleaning procedure as long as the $\mathrm{Rh}$ mirror integrity is ensured. Other experiments in this direction are foreseen.

\section{Conclusions}

This paper presents an experimental investigation of the laser cleaning of tokamak diagnostic FMs with laboratory-scale facilities. By means of PLD, Rh films functional as FMs and $\mathrm{C}$ contaminants with selected properties were produced. The same laser source was exploited to remove the $\mathrm{C}$ deposits from the $\mathrm{Rh}$ films surface thanks to the laser cleaning technique. We deposited two peculiar C morphologies (compact film and porous "quasi-film") representative of those of the wide variety of $\mathrm{C}$ contaminants found in tokamaks. A sample handling procedure was developed to clean relevant areas (some $\mathrm{cm}^{2}$ ) in a reasonable time (few minutes). Laser cleaning was performed by means of NIR and UV laser pulses, both separately and combined, choosing the other process parameters in order to preserve $\mathrm{Rh}$ films integrity. Considering the compact $\mathrm{C}$ contamination, $\mathrm{R}_{\text {spec }}$ was fully recovered with a single NIR scan. The same procedure applied to the porous $\mathrm{C}$ did not determine a complete $\mathrm{R}_{\text {spec }}$ recovery in the visible and UV region, not even after further NIR scans. UV cleaning trials gave comparable results in terms of $\mathrm{R}_{\text {spec }}$ recovery. The best results were obtained with a NIR scan followed by a UV one. A significant $R_{\text {spec }}$ recovery on 260-1800 $\mathrm{nm} \lambda$ range was achieved. The effectiveness of the approach here discussed in producing and removing different $\mathrm{C}$ morphologies from $\mathrm{Rh}$ films was proven. Further experiments are foreseen to extend the investigation to ITERrelevant contaminants, like tungsten and beryllium proxy (due to Be toxicity) deposited onto other FMs candidate materials (e.g. molybdenum).

\section{Acknowledgments}

The authors wish to thank G. Granucci, G. Grosso, M. Lontano, B. Eren, L. Marot and E. Meyer. Part of this work is supported by the European Communities under the contract of Association EURATOM/ENEA-CNR. The views and opinions expressed herein do not necessarily reflect those of the European Commission.

\section{References}

[1] E. E. Mukhin et al., Nucl. Fusion 52 (2012) 013017.

[2] A. Widdowson et al., J. Nucl. Mater. 415 (2011) 1199.

[3] M. Wisse et al., Fus. Eng. Des. 89 (2014) 122.

[4] Y. Zhou et al., J. Nucl. Mater. 415 (2011) 1206

[5] A. Uccello et al., Fus. Eng. Des. 88 (2013) 1347.

[6] M. Passoni et al., J. Nucl. Mater. 404 (2010) 1.

[7] A. Uccello et al., J. Nucl. Mater. (2013) 261.

[8] T. Sugie et al., 12th Meeting of the ITPA TG on Diagnostics, (2007).

[9] H. Roche et al., Phys. Scripta T138 (2009) 014028.

[10] C. H. Skinner et al., Fus. Sci. and Tech. 64 (2012) 1.

[11] M. Mayer et al., Phys. Scripta T111 (2004).

[12] M. Richou et al., Carbon 45 (2007) 2723.

[13] Y. Raitses et al., J. Nucl. Mater. 375 (2008) 365369.

[14] S. I. Krasheninnikov et al., Plasma Phys. Contr. Fus. 53 (2011) 083001.

[15] A. Zani et al., Carbon 56 (2013) 358.

[16] A. C. Ferrari et al., Phys. Rev. B 61 (2000) 14095107.

[17] J. Robertson, Mater. Sci. Eng. R. 37 (2002) 12928.

[18] V. I. Merkulov et al., Appl. Phys. Lett. 73 (1998) 2591.

[19] M. Wisse et al., Fus. Eng. Des. 88 (2013) 388. 